\title{
Reduction of Air Ion Mobility to Standard Conditions
}

\author{
H. Tammet \\ Department of Environmental Physics, University of Tartu \\ 18 Ülikooli Str., Tartu, EE2400, Estonia \\ Internet: Hannes.Tammet@ut.ee
}

\begin{abstract}
The Langevin rule of the reduction of ion mobilities is not correct when applied to atmospheric ions. The StokesMillikan equation provides an alternative valid in the mobility range of large air ions. A new semiempirical model combines the kinetic theory and Stokes-Millikan equation in the full range of air ion mobilities. The errors caused by the usage of the Langevin rule are discussed.
\end{abstract}




\section{Introduction}

The Langevin rule:

$$
K_{\text {reduced }}=K_{\text {measured }} \frac{273.15 \mathrm{~K}}{T} \frac{p}{101325 \mathrm{~Pa}} .
$$

A warning by MASON: the Langevin rule is correct only in the limit of zero-size ions and considerable errors are possible when applying the rule to the cluster ions (the warning has been neglected in atmospheric electricity).

The mobilities of large air ions have been presented as measured, and a question about reduction has not been raised.

How to process the data about intermediate air ions? They are a subject of active research today.

How to measure the deviation from the Langevin rule?

A suggestion: fit the temperature and pressure variation of the mobility with an empiric equation

$$
K=\mathrm{const} \frac{T^{\tau}}{p^{\psi}} .
$$

where $\tau$ and $\psi$ should equal 1 if the Langevin rule is correct.

When the temperature and pressure variation of the mobility is given in some different way, the parameters $\tau$ and $\psi$ are estimated:

$$
\tau=\frac{\mathrm{d} K}{\mathrm{~d} T} \frac{T}{K}, \quad \psi=-\frac{\mathrm{d} K}{\mathrm{~d} p} \frac{p}{K} .
$$

The deviations of the parameters $\tau$ and $\psi$ from the value 1 are the characteristics of the error of the Langevin rule.

If the temperature and pressure variation of the mobility does not follow the power law, Equation (2) is interpreted as an approximation valid in a narrow temperature and pressure range. In this occasion the values of $\tau$ and $\psi$ determined according to Equation (3) depend on the temperature and pressure in the wide temperature and pressure range.

Experimental complication: the temperature variation of the ion chemical composition obscures the physical effect (e.g. Hõrrak et al., 1994). 


\section{Langevin model and the kinetic theory}

Chapman-Enskog kinetic theory (a presumption: the free molecule regime):

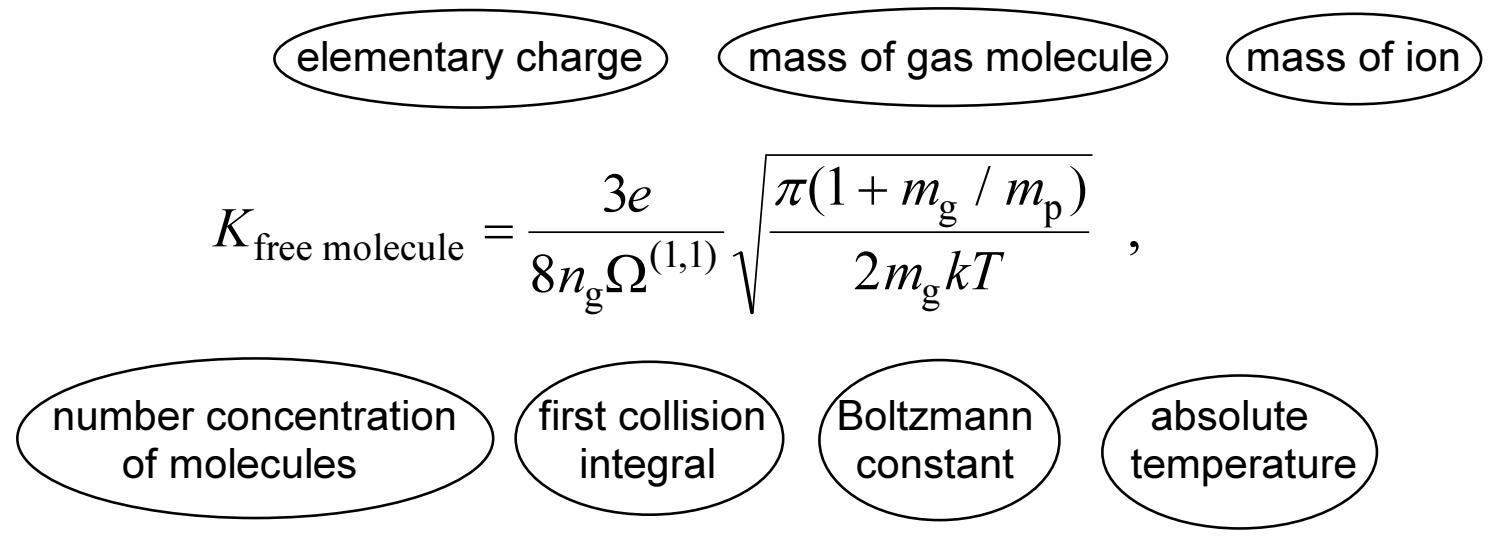

In the Langevin model an ion is considered as a point mass carrying one elementary charge, and the interaction between the ion and the gas molecule separated by the distance $r$ is described by the potential of induced dipole

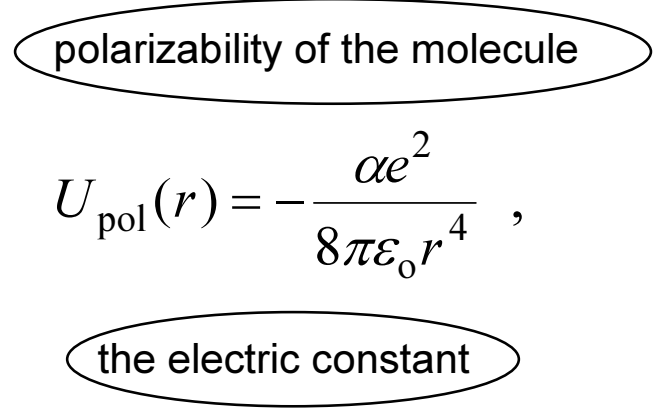

The geometric sizes of the colliding particles is ignored.

The resulting expression of the mobility is

$$
K_{\text {Langevin }}=0.5105 \frac{e}{n_{\mathrm{g}}} \sqrt{\frac{\varepsilon_{\mathrm{o}}\left(1+m_{\mathrm{g}} / m_{\mathrm{p}}\right)}{\alpha m_{\mathrm{g}}}} .
$$

It follows the air ion mobility is inversely proportional to the air density and the values of the parameters of Equation (2) are $\tau=\psi=1$. 


\section{Stokes-Millikan model}

Large air ion mobilities (Stokes, Cunningham, Knudsen, Weber, Millikan):

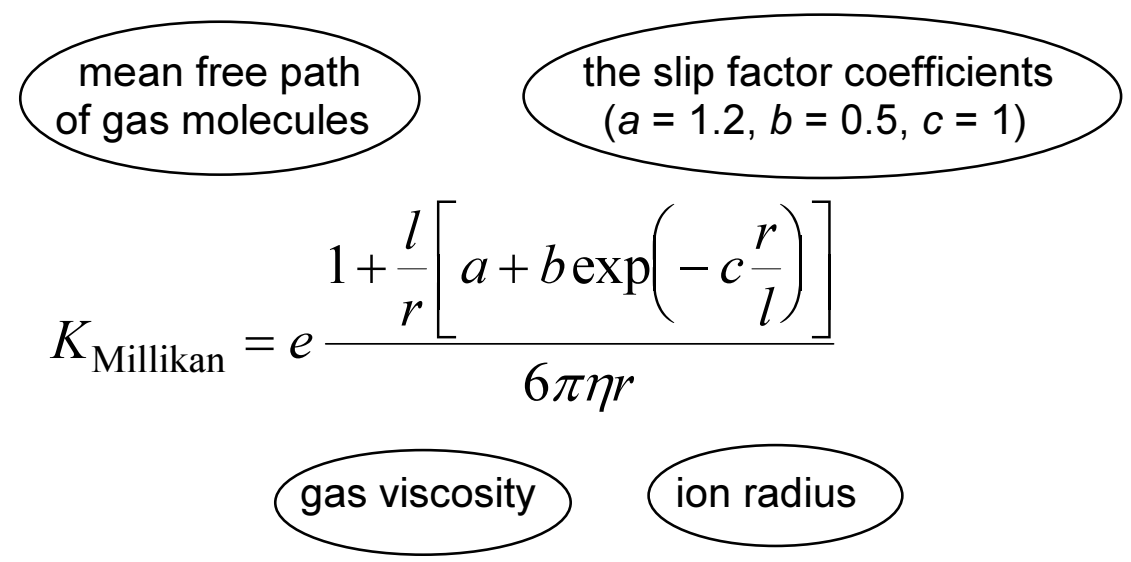

Approximate equation

$$
\eta=0.192(T: \mathrm{K})^{0.8} \mu \mathrm{Pa} \mathrm{s},
$$

(relative deviation from the Sutherland approximation $< \pm 0.2 \%$ in temperature interval $-50^{\circ} \ldots+50^{\circ} \mathrm{C}$ ).

Big particle limit $\tau=-0.8$ and $\psi=0$ is very far from the Langevin model.

Air ions are never very big particles in the sense of the slip factor. The Knudsen number $l / r$ is reaching a value of 1 at the mobility less than $0.0002 \mathrm{~cm}^{2} \mathrm{~V}^{-1} \mathrm{~s}^{-1}$.

The fine particle limit:

$$
K_{\text {Millikan } \rightarrow 0}=\frac{e(a+b) l}{6 \pi \eta r^{2}} \quad \frac{l}{\eta}=\frac{1.256}{p} \sqrt{\frac{k T}{m}} .
$$

Result $\tau=0.5$ and $\psi=1$.

The pressure variation is the same as in the Langevin model, but the temperature variation is different.

The limit (9) does not produce correct values for small ion mobilities (Annis et al., 1972). The Stokes-Millikan equation neglects polarization interaction and assumes inelastic scattering of molecules by particles that is not true in the molecular size range. 


\section{Semiempirical model}

The semiempirical model (Tammet, 1995) is approaching the kinetic theory Equation (4) in the limit of zero size and the Stokes-Millikan equation (7) for big particles. It is equally valid for small, intermediate and large air ions. The collision distance is considered as a function of the interaction energy. The polarization interaction is included according to the $(\infty-4)$ potential model. The dependence of the law of the reflection of gas molecules on the particle size is considered using the Einstein model of "melting" the particle internal energy degrees of freedom. The model consists of three parameters that cannot be evaluated in theory. The values of these parameters

the internal density of particle matter $\rho=2.07 \mathrm{~g} \mathrm{~cm}^{-3}$

the extra distance added to the sum of ion and molecule radii $h=0.115 \mathrm{~nm}$

the critical radius of the transfer from elastic to inelastic collisions $r_{\mathrm{cr}}=1.24 \mathrm{~nm}$ are estimated fitting the model to the old mass-mobility data by Kilpatrick (1971). The Kilpatrick's data have been discussed and confirmed by various authors (e.g. Meyerott et al., 1980, Böhringer et al., 1987), and there is no better data set available today.

The semiempirical model cannot be expressed by an analytic expression. It is expressed as a Pascal-function* in the paper (Tammet, 1995). The algorithm of air ion mobility reduction according to the model consists of two stages: first, the particle size should be calculated at a given temperature and pressure and then the reduced mobility should be calculated according to the size, standard temperature and standard pressure. The Pascal-statement where the Pascal-functions published in (Tammet, 1995) are used is:

\section{ReducedMobility :=}

1.602 * Mobility $(28.96,0.00171,0.3036,44,0.8,1013.25,273.15,2.07,1$, MassDiameter (millibar, 273.15 + Celsius, 2.07, 1, MeasuredMobility / 1.602));

The mobilities are expressed in $\mathrm{cm}^{2} \mathrm{~V}^{-1} \mathrm{~s}^{-1}$.

\footnotetext{
*An erratum should be corrected in the Pascal-function Mobility (Tammet,1995): replace if $\mathrm{Kn}<0.03$ \{underflow safe\} then $y:=1$ else $y:=\exp (-c / K n)$; with if $\mathrm{Kn}<0.03$ \{underflow safe\} then $y:=0$ else $y:=\exp (-c / \mathrm{Kn})$;
} 


\section{Discussion}

The temperature and pressure variation of parameters $\tau$ and $\psi$ is illustrated in Figure 1. The character of variation is different for the air ions of different mobility. A variation of the pressure factor $\psi$ is relatively simple. It will differ from the Langevin value 1 in the range of large air ions due to the decrease in the role of the slip factor with the decrease in Knudsen number. The macroscopic limit of $\psi$ is zero but the real values of $\psi$ for large air ions are far from this limit.

The variation of the temperature factor $\tau$ does not follow simple rules because the temperature effect is influenced by many different physical factors.
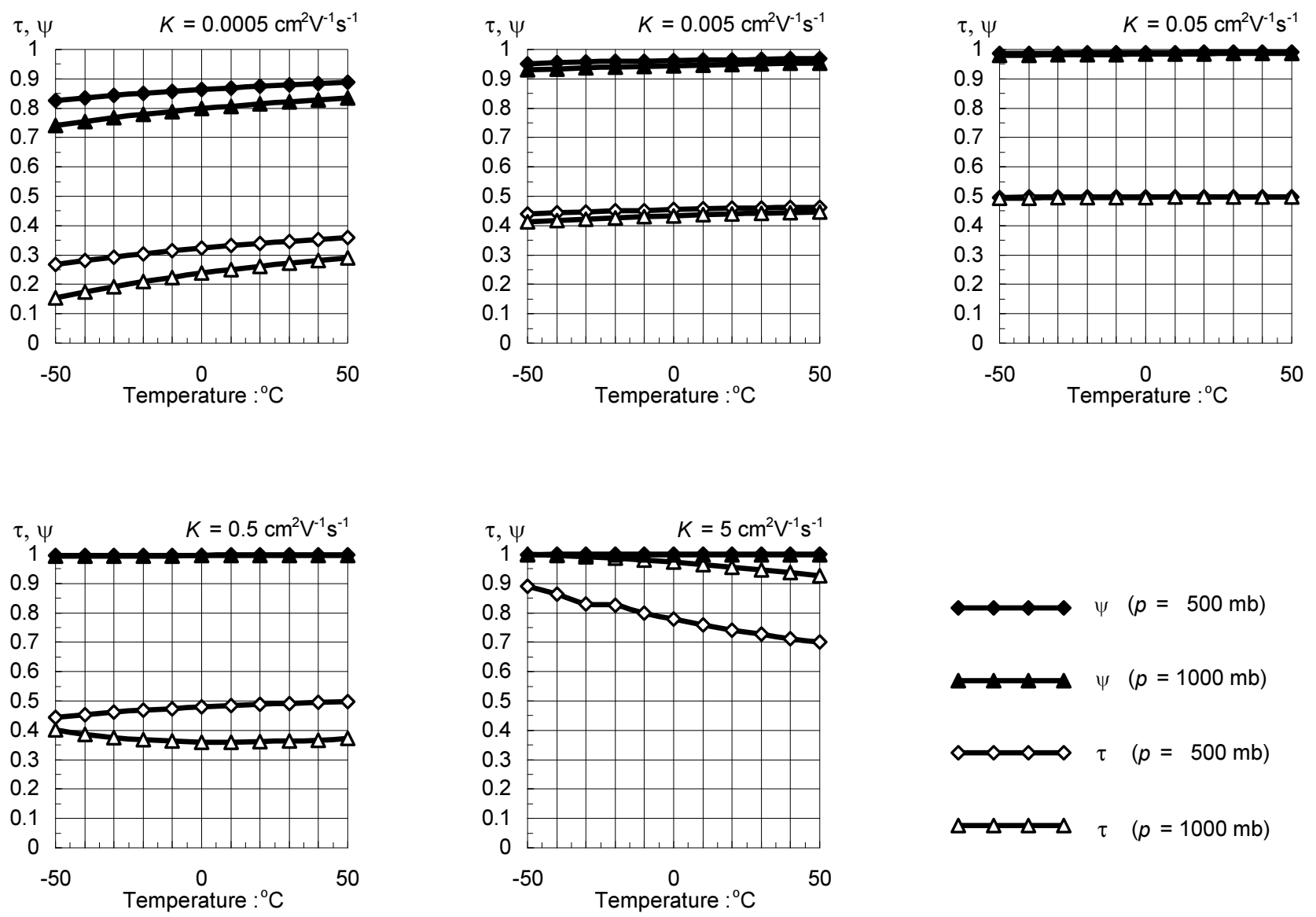

Figure 1. Temperature and pressure variation of parameters $\tau$ and $\psi$ for the air ions of various mobility. 
The dependence of the parameters $\tau$ and $\psi$ on the air ion mobility is shown in Figure 2. The value of $\tau$ is far from the Langevin value 1 even in case of small atmospheric ions, typical mobility of which is about $1.5 \mathrm{~cm}^{2} \mathrm{~V}^{-1} \mathrm{~s}^{-1}$. The Langevin model could be considered as a fair approximation only in case of extremely high mobilities characteristic of atomic and pure molecular ions created in some laboratory experiments.

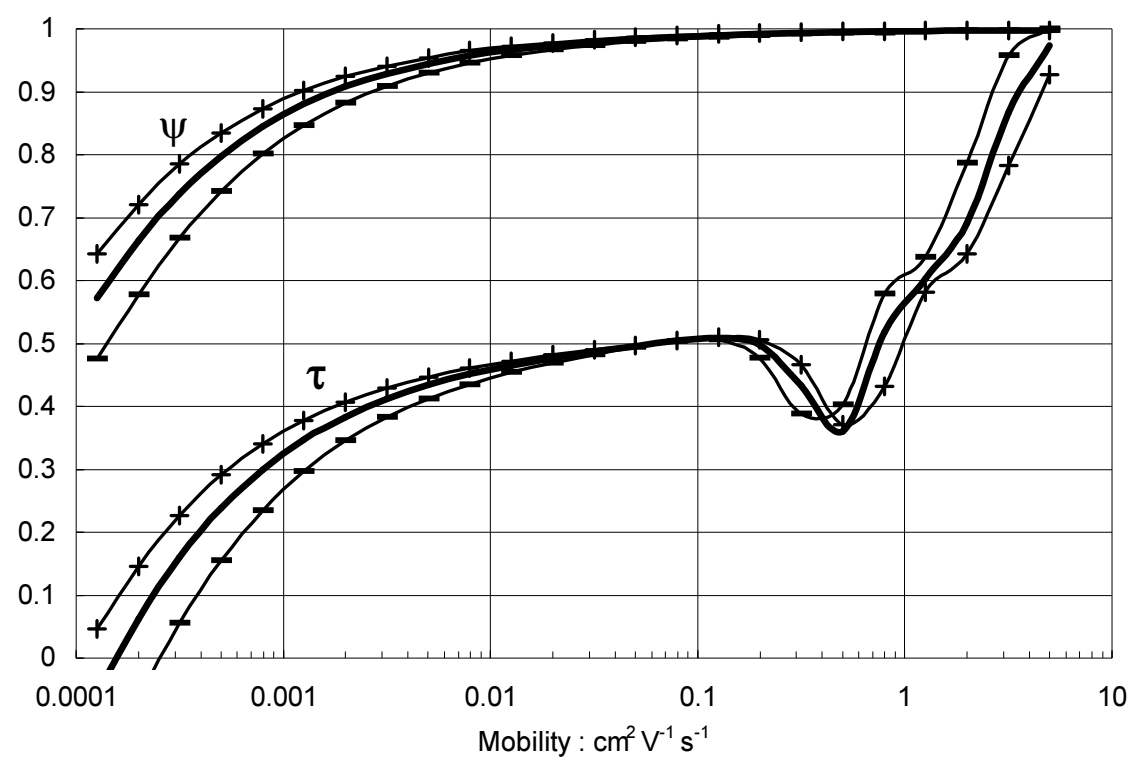

Figure 2. Air ion mobility temperature and pressure variation parameters $\tau$ and $\psi$ at pressure $1000 \mathrm{mb}$. The unmarked curves correspond to temperature $0^{\circ} \mathrm{C}$, the curves marked with + and - correspond to temperatures $+50^{\circ} \mathrm{C}$ and $-50^{\circ} \mathrm{C}$. 
The weakness of the Langevin rule is shown by an example. The air ion massmobility correlation in the mass interval of 35-2122 u has been measured by Kilpatrick (1971) at $200^{\circ} \mathrm{C}$, and the mobilities have been published as reduced to standard conditions using the Langevin rule. The fitting of these data yields a regression equation

$$
K \approx\left(\sqrt[3]{\frac{850 \mathrm{u}}{m}}-0.3\right) \mathrm{cm}^{2} \mathrm{~V}^{-1} \mathrm{~s}^{-1} \quad m \approx \frac{850 \mathrm{u}}{\left(0.3+K: \mathrm{cm}^{2} \mathrm{~V}^{-1} \mathrm{~s}^{-1}\right)^{3}}
$$

published in CRC Handbook, 1993.

In the paper (Tammet, 1995) the original $200^{\circ} \mathrm{C}$ data were restored and the mobilities at $0^{\circ} \mathrm{C}$ were recalculated according to the new model. Now the best fit is achieved at different coefficients of the equation

$$
K \approx\left(\sqrt[3]{\frac{1200 \mathrm{u}}{m}}-0.2\right) \mathrm{cm}^{2} \mathrm{~V}^{-1} \mathrm{~s}^{-1} \quad m \approx \frac{1200 \mathrm{u}}{\left(0.2+K: \mathrm{cm}^{2} \mathrm{~V}^{-1} \mathrm{~s}^{-1}\right)^{3}}
$$

The ratio of air ion masses 1200/850 estimated according to different approximations is too big to be neglected.

The values of parameters in the size-mobility model can be justified after improved experimental data of mass-mobility or size-mobility correlation becomes available, and advanced models can be proposed. If the measurements are published as reduced to standard conditions according to a certain model, a correction of the data could be required in the future. Thus, the actual values of mobility completed by the values of temperature and pressures are recommended for publication. If only the reduced mobilities are published, the algorithms of reduction and restoration of original data should be indicated.

Acknowledgment. This study was supported by the Estonian Science Foundation Grant no. 1226 and US Government Fulbright Grant no. 19952. 


\section{References}

Annis, B.K., Malinauskas, A.P., and E.A. Mason, Theory of drag on neutral or charged spherical aerosol particles. J. Aerosol Sci. 3, 55-64, 1972.

Böhringer, H., Fahey, D.W., Lindinger, W., Howorka, F., Fehsenfeld, F.C., and D.L. Albritton, Mobilities of several mass-identified positive and negative ions in air. Int. J. Mass Spectrom. Ion Processes 81, 45-65, 1987.

Chapman, S. and T.G. Cowling, The mathematical theory of non-uniform gases. Cambridge University Press, Cambridge, 1970.

CRC Handbook of Physics and Chemistry, 74th Edition, CRC Press, Boca Raton, Section 14, 25-26, 1993.

Hõrrak, U., Iher, H., Luts, A., Salm, J., and H. Tammet, Mobility spectrum of air ions at Tahkuse Observatory. J. Geophys.Res. D 99, 10697-10700, 1994.

Hutchins, D.K, M.H. Harper, and R.L. Felder, Slip correction measurements for solid spherical particles by modulated dynamic light scattering, Aerosol Sci. Technol., 22, 202-218, 1995.

Kilpatrick, W. D., An experimental mass-mobility relation for ions at atmospheric pressure, in Proc. 19th Annu. Conf. Mass Spectrosc., 320-325, 1971.

Mason, E.A., Ion mobility: its role in plasma chromatography. In Plasma Chromatography (Edited by T.W. Carr), 43-93. Plenum Press, New York and London, 1984.

Mason, E.A. and E.W. McDaniel, Transport properties of ions in gases, John Wiley, New York, 1988.

Meyerott, R.E, Reagan J.B., and R.G. Joiner, The mobility and concentration of ions and the ionic conductivity in the lower stratosphere. J. Geophys. Res. A 85, 1273-1278, 1980.

Tammet, H., Size and mobility of nanometer particles, clusters and ions, J. Aerosol Sci. 26, 459-475, 1995.

Thuillard. M., Electric mobility measurements of small ions in the temperature range $-40-20^{\circ} \mathrm{C}$ at constant relative humidity of $87 \%$, J. Aerosol Sci. 26, 219$225,1995$. 